\title{
An Assisted Positioning System based on Low Energy Bluetooth and Six-axis Accelerometer
}

\author{
Siquan $\mathrm{Hu}^{1, \mathrm{a}}$, Jingyue Liu ${ }^{1, \mathrm{~b}}$ and Chundong She $\mathrm{S}^{2, \mathrm{c}}$ \\ ${ }^{1}$ School of Computer and Communication Engineering,University of Science and Technology \\ Beijing, Beijing, China \\ ${ }^{2}$ School of Electronic Engineering, Beijing University of Posts and Telecommunications, Beijing, \\ 100876, China \\ ahusiquan@ustb.edu.cn, b862544022@qq.com, cshurcd@vip.sina.com
}

Keywords: BLE ; CC254x ;Six-axis Accelerometer; Assisted Positioning.

Abstract. We implement an assisted positioning system, based on BLE and six-axis accelerometer that includes an accelerometer and a gyroscope. The system uses two on-chip CC254x with BLE features for wireless transmission and MATLAB for the data processing in the cloud. After quadratic integral of acceleration data, we get displacement of the moving object. After integral of angular acceleration data, we get rotation angle of the moving object. According to the principle of different coordinate system conversion, combining the displacement and rotation angle, we get the trajectory of the moving object. When applied to GPS signal blocked areas, the system can implement assisted positioning.

\section{Introduction}

Nowadays, more and more enterprises and individual have created a demand of positioning technology in our daily life such as car navigation, livestock, monitoring of the particular patient. GPS navigation and positioning technology in most cases can effectively solve the problem. However in certain areas such as tunnels, buildings, interior, GPS signal are blocked under certain weather condition, the signal will become very weak and be hard to get position.

There are many wireless communication technologies currently on the market, such as ANT, ZigBee, Bluetooth, Taking the market share and compatibility into account, selecting Bluetooth as wireless communication means is undoubtedly a wise choice. Low power consumption has become a significant feature of BLE. This features makes BLE widely used in short range communication and attracts a lot of fans and attention of investors which have invested in human and material resources to the development and application of BLE.

Now the market's six-axis accelerometer basically consists of a accelerometer and a gyroscope . The accelerometer get acceleration changes of three axes and the gyroscope obtain angular velocity changes around three axes. While the GPS navigation technology has become more mature, along with transparency of information, the positioning technology has also become more and more important where GPS signal are blocked in some targeted areas or weak signal regions. Based on this, the authors design and implement a assisted positioning system, which processing data in the cloud.

\section{System design and analysis}

System Hardware. Hardware block of the system consists of two parts .One is the Mobile Facility consisting of a six-axis accelerometer and a on-chip CC254x with BLE features, the other is Data Processing Facility including a on-chip CC254x with BLE features and a PC[1,2].

Six-axis accelerometer. The six-axis accelerometer consists of an accelerometer and a gyroscope. The data six-axis accelerometer exporting has been through Kalman Filter which effectively improves the system accuracy.

Microcontroller. CC2541F256 is our main processor .It is a power-optimized SoC for BLE applications made by TI. It has an enhanced 8051 MCU. There have 256 Kbytes of embedded Flash 
memory, 8 Kbytes of embedded SRAM including 12-bit ADCs, I2C, GPIO, UART and a BLE RF transceivers supporting data transfer rate of $250 \mathrm{kbps}, 500 \mathrm{kbps}, 1 \mathrm{Mbps}, 2 \mathrm{Mbps}[3]$.

Firmware Design. TI BLE Stack provides more layers than Basic BLE stack such as Application, GAP Profiles and GATT Profiles. The GAP Profile defines the pair connection of a master and a slave. The GATT profile defines the format of data that contains the acceleration data and angular acceleration data [4].

Wireless Transmission. There are there steps for data transmission between the Mobile Facility and Data Processing Facility.

Establishing Connection. The CC2541F256 in the Mobile Facility plays a role as a master; the CC2541F256 in the Data Processing Facility plays a role as a slave. Before transporting the data, there should establish a connection .we use white list mechanism when establish it [5].

Collecting Data. After the connection is established, we use the six-axis accelerometer to measures and records acceleration data and angular acceleration data of a moving object. The master uses DMA mode to read the data through the serial port.

Transporting Data. The master writes 18 bytes to the value of the characteristic that the GATT profile defines each time and sends the values to the slave by transceiver of the master. The function GATT_WriteLongCharValue() will be used.

When the slave in the Data Processing Facility receives the data, the function HalUARTWrite() will be used to process the data. Finally the slave transports the data to the PC.
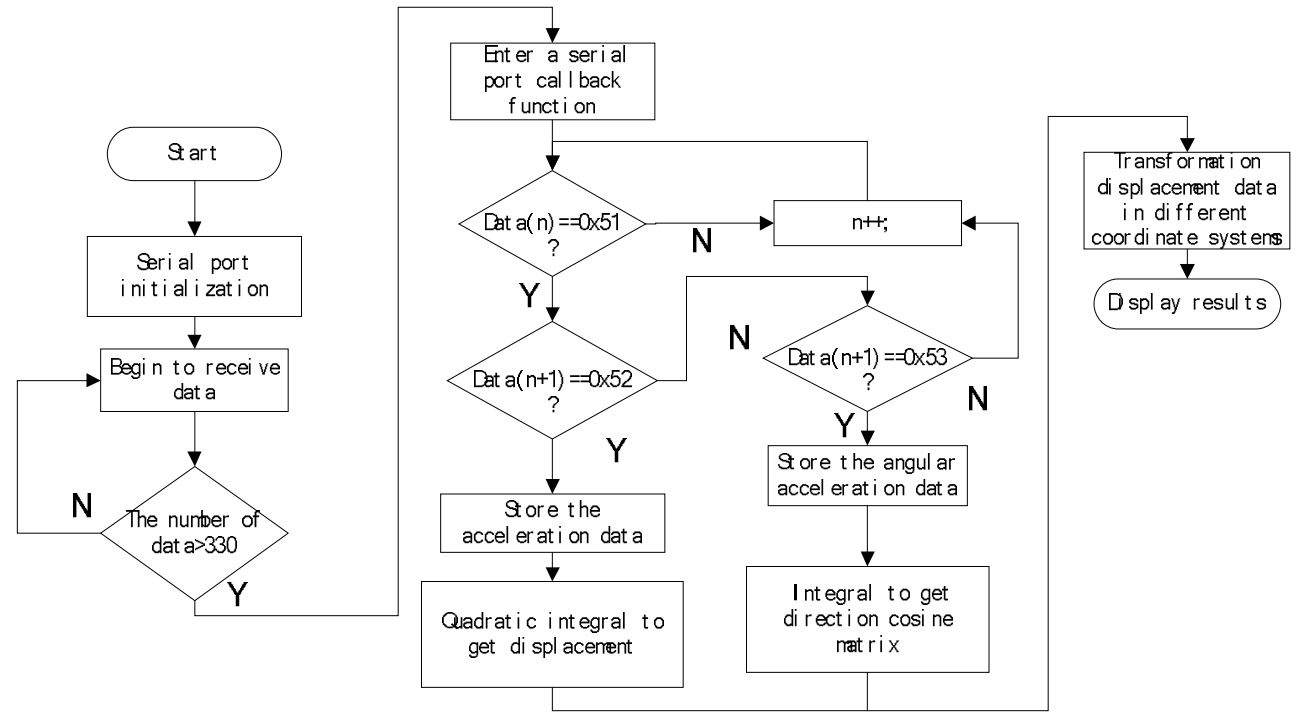

Fig .1 The software flow chart

Positioning Algorithm In the Data Processing Facility we use MATLAB to process the data [4]. Fig. 1 shows the software flow chart. When acceleration data is through quadratic integral in the unit time, we get the displacement of the object. When angular acceleration data is through integral in the unit time, we get the rotation angle of the moving object. We can get the direction cosine matrix in the unit time according to the rotation angle. The displacement multiplied by the direction cosine matrix, we get the trajectory of the object.

Integral method. Generally, there are two kinds of integral methods, one is time domain integral, the other is the frequency domain integral. The time domain integral method is more suitable for application in this example. Numerical integration method contains Trapezoidal formula, Compound trapezoid formula, the rectangle formula, Simpson formula and Newton - cotes formulas. Due to the complex trapezoidal not only meets the requirements of the experiment, but also is easy to operation. We adopted the complex trapezoidal integral formula [6]. Symbols $a(j)$ said acceleration, where $j=1,2, \ldots \ldots n$. According to compound trapezoid formula, speed signal is: 


$$
v(j)=v(j-1)+\sum_{i=1}^{n-1}[a(i)+a(i+1)] /\left(2 f_{s}\right) j=1,2, \ldots \ldots, n-1, v(0)=0
$$

Displacement calculation expression is:

$$
s(k)=v(k-1)+\sum_{i=1}^{n-1}[v(i)+v(i+1)] /\left(2 f_{s}\right), k=1,2, \ldots \ldots, n-1, s(0)=0 .
$$

Different coordinate systems conversion principle. In the field of mathematics, the different coordinate systems transformation operations would involve a matrix to represent their relative position. We set two Cartesian coordinate systems, one is $\mathrm{OX}_{1} \mathrm{Y}_{1} \mathrm{Z}_{1}$, and the other is $\mathrm{OX}_{2} \mathrm{Y}_{2} \mathrm{Z}_{2}$. Fig. 2 shows the relationship between the coordinate system $\mathrm{OX}_{1} \mathrm{Y}_{1} \mathrm{Z}_{1}$ and $\mathrm{OX}_{2} \mathrm{Y}_{2} \mathrm{Z}_{2}$.

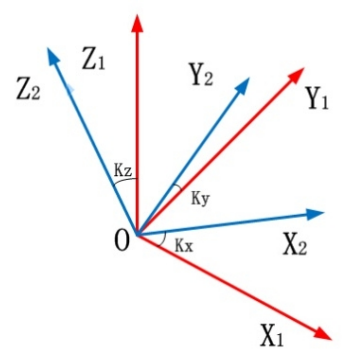

Fig.2 The relationship between the coordinate

$\mathrm{K}_{\mathrm{x}}, \mathrm{Ky}$ and $\mathrm{Kz}$ are respectively the three axis angles while the coordinate $\mathrm{OX}_{1} \mathrm{Y}_{1} \mathrm{Z}_{1}$ rotating from $\mathrm{OX}_{2} \mathrm{Y}_{2} \mathrm{Z}_{2}$.

When three Euler Angle $\mathrm{K}_{\mathrm{x}}, \mathrm{Ky}$ and $\mathrm{Kz}$ are all small angles, the direction cosine matrix can be expressed as:

$$
C_{2}^{1}=\left[\begin{array}{ccc}
1 & K_{Z} & -K_{Y} \\
-K_{Z} & 1 & K_{X} \\
K_{Y} & -K_{X} & 1
\end{array}\right]
$$

\section{Implementation and Test Results}

In order to test the accuracy of the system, we have tested it in two situations:

Uniformly accelerated linear motion we put the Mobile Facility of the system on a car that run in a uniformly accelerated linear motion with the direction of the original coordinate system. The whole testing time is $95 \mathrm{~s}$, the first $20 \mathrm{~s}$, the car was still, the remaining $75 \mathrm{~s}$, the car run in uniformly accelerated linear motion. The actual displacement of the car moved is $50 \mathrm{~m}$, the results of the testing showed is $49 \mathrm{~m}$. Fig. 3 shows the speed and Fig. 4 shows the displacement of the car in this test.

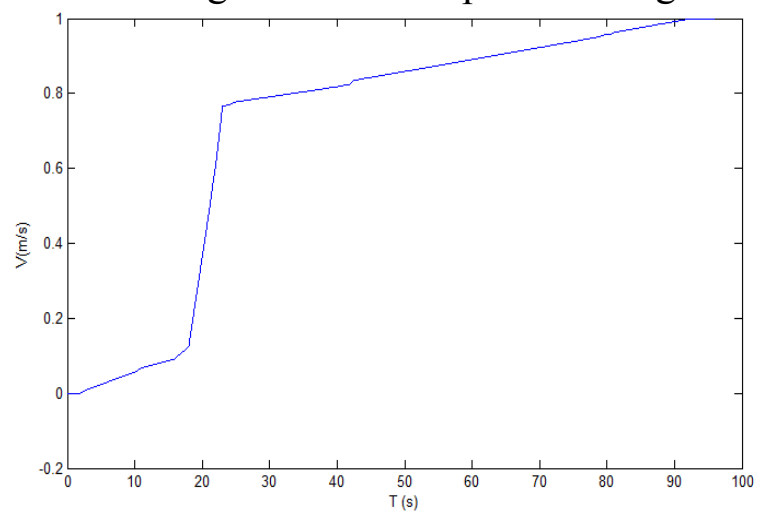

Fig.3 The speed of the car

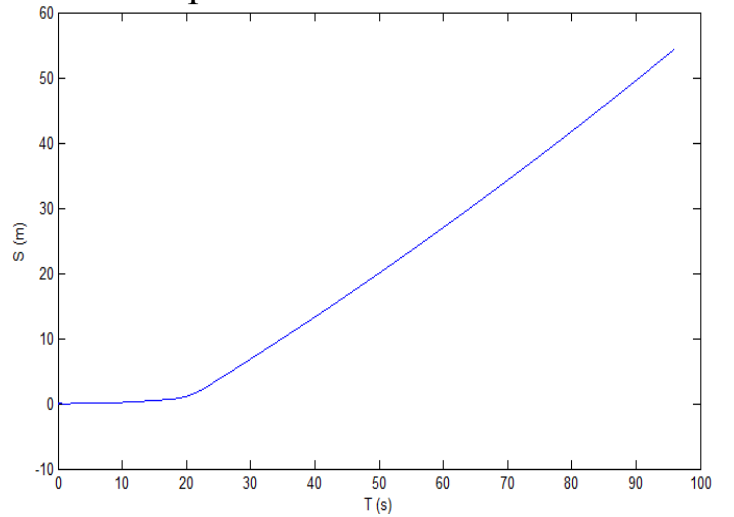

Fig.4 The displacement of the car

Curvilinear motion People who carry the Mobile Facility of the system do curvilinear motion, the direction of the people moves is 45 degrees with the $\mathrm{X}$-axis direction of original coordinate system . 
the actual distance between the start end and the stop end is $71 \mathrm{~m}$, the results of the experiment shows is $73 \mathrm{~m}$.The degrees of the results shows is 58.9 degrees with the $\mathrm{X}$-axis direction of original coordinate system . The Fig. 5 shows the speed and Fig. 6 shows trajectory of the people in testing.

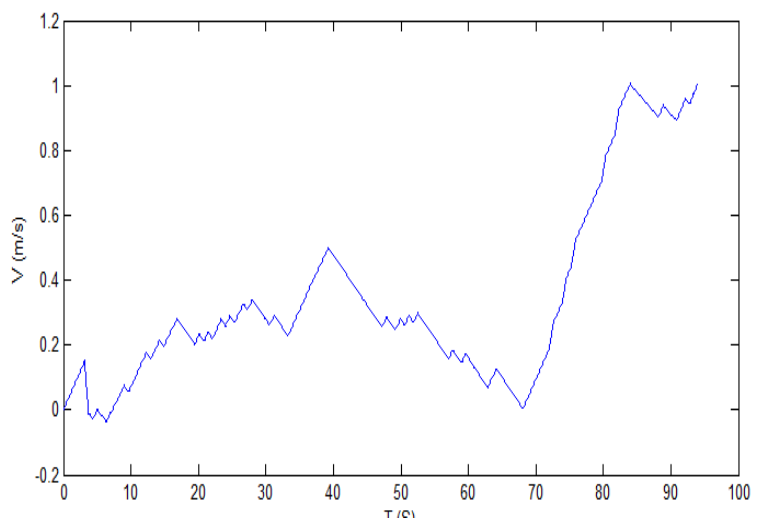

Fig.5 The speed of the people

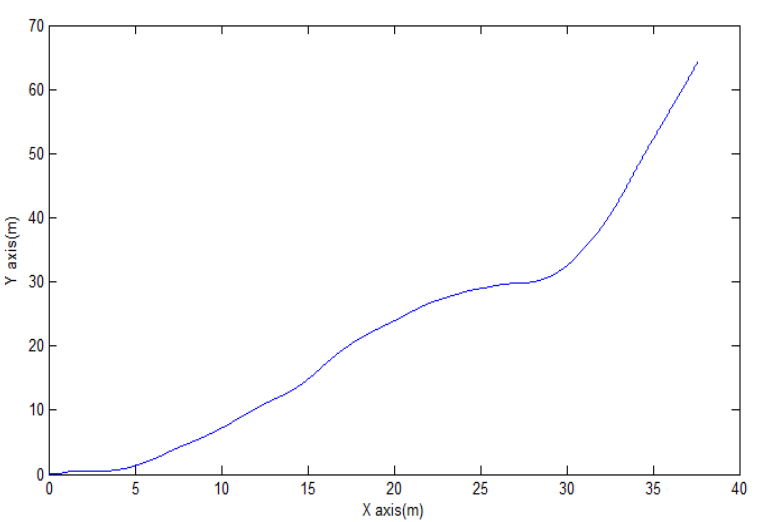

Fig.6 The trajectory of the people

\section{Conclusion}

We have successfully designed and implemented the assisted positioning system, based on BLE and six-axis accelerometer, including hardware device and software algorithm.

Using the wireless transmission of BLE and MATLAB to process the data we can obtain the trajectory of the moving object finally. After Repeated test, we get the accuracy of the system, the error of a linear motion is $2 \%-10 \%$, the displacement error of a curvilinear motion is $5 \%-16 \%$.the direction error of a curvilinear motion is 10degrees-20 degrees. The system can be applied in GPS blocked area as a useful assisted positioning mechanism.

\section{Acknowledgements}

The study was supported by the National Natural Science Foundation of China (No. 91438120) and the Foundation of Key Laboratory of Aerospace Broadband Network Technology.

\section{References}

[1] Zhe-Min Lin, Cheng-Hung Chang, Nai-Kuan Chou, Yuan-Hsiang Lin, Bluetooth Low Energy (BLE) Based Blood Pressure Monitoring System,J.Intelligent Green Building and Smart Grid (IGBSG),IEEE, ernational conference .

[2] B. Yu, L. Xu, and Y. Li,Bluetooth Low Energy Based Mobile Electrocardiogram Monitoring System, in Proc. of IEEE Int. Conf. on information and automation, pp. 763-767, Jun., 2012

[3] Information on http://www.ti.com/lit/ug/swru271b/swru271b.pdf

[4] Information on http://www.bluetooth.com/Pages/SIGMembership.aspx

[5] Ji Wang,Xiao Hu. MATLAB in Vibration Signal Processing ,Bei Jing,2006, In Chinese

[6] Xinglong Wang, Inertial navigation foundation, Bei Jing,2013,In Chinese 